\title{
Erratum
}

\section{Degenerate elliptic systems and applications to Ginzburg-Landau type equations, Part I}

\author{
Zheng-Chao Han, YanYan Li \\ Department of Mathematics, Rutgers University, New Brunswick, NJ 08903, USA
}

The following paragraph was added by the authors in the galley proof to our paper, "Degenerate elliptic systems and applications to Ginzburg-Landau type equations, Part I", Calc. Var. 4, 171-202 (1996). Unfortunately, it was not printed in the paper due to production error.

Note added in proof: After completing our work, we learned that M.C. Hong recently completed a preprint, "Asymptotic Behavior for Minimizers of a GinzburgLandau-Type Functional in Higher Dimensions Associated with n-Harmonic Maps", where he proves results related to some part of our paper, namely, Theorem 0.2 of our paper. More specifically, Hong's paper establishes weak convergence in $W_{\text {loc }}^{1, n}\left(\bar{\Omega} \backslash\left\{a_{1}, a_{2}, \cdots, a_{|d|}\right\} ; \mathbf{R}^{\mathbf{n}}\right)$ for a sequence of selected minimizers, obtained through a regularization procedure, of the Ginzburg-Landau functional (see his Theorem 1.2), while Theorem 0.2 of our paper establishes strong convergence in $W_{\operatorname{loc}}^{1, n}\left(\bar{\Omega} \backslash\left\{a_{1}, a_{2}, \cdots, a_{|d|}\right\} ; \mathbf{R}^{\mathbf{n}}\right)$ and in $C_{\operatorname{loc}}^{0}\left(\bar{\Omega} \backslash\left\{a_{1}, a_{2}, \cdots, a_{|d|}\right\} ; \mathbf{R}^{\mathbf{n}}\right)$ for a sequence of any minimizers of the Ginzburg-Landau functional. 